\title{
Cleveland
}

Clinic

\section{Body Weight Changes In Patients With Active Cushing's Disease After Transsphenoidal Surgery}

Philip C Johnston ${ }^{1}$, Laurence Kennedy ${ }^{1}$, Amir Hamrahian ${ }^{1}, \mathrm{Jim}_{\text {Bena }}{ }^{2}$, Robert Weil ${ }^{3}$

Dept of Endocrinology, Diabetes and Metabolism ${ }^{1}$ and Biostatistics ${ }^{2}$, Cleveland Clinic , OH, USA

Dept of Neurosurgery, ${ }^{3}$ Geisinger Health System, Danville, PA, USA

\section{INTRODUCTION}

- Cushing's disease (CD) is usually associated with alterations in body mass index. Successful pituitary surgery can result in long term remission with sustained weight loss.

- We examined rates of body weight change (in the post-operative period within 6 months) in patients with active CD who underwent transsphenoidal surgery (TSS) and the relationship of weight loss to remission status.

\section{METHODS}

- Clinical data was obtained from a CD database in addition to an online patient medical record (EPIC).

- All patients with biochemically confirmed active CD underwent their first TSS by a single neurosurgeon at the Cleveland Clinic: (October 2004 to August 2013).

- None of the patients received glucocorticoids during surgery.

- Initial remission was defined by nadir cortisol $<5$ $\mu \mathrm{g} / \mathrm{dl}$ and/or ACTH $<5 \mathrm{pg} / \mathrm{ml}$ within the immediate post-operative period ( $72 \mathrm{hrs}$ ).

- Long term remission ( $\geq 12$ months) was defined as $24 \mathrm{hr}$ UFC < ULN (upper limit of normal), and/or sequential midnight salivary cortisols $<U L N$, and $1 \mathrm{mg}$ DST; 8 am cortisol $<1.8 \mu \mathrm{g} /$ dl.

- Statistical analyses were performed using SAS software (Version 9.2;Cary, NC).

\section{RESULTS}

- Data was available for 88 patients (F:63,M:25), mean age at presentation was 47 years (range 24-87 years), median follow up 52 months (12-118 months). - No significant differences in baseline demographics including pituitary adenoma size $(p=0.25)$ and $B M I$ $(p=0.21)$ were observed between the two groups.

- 64 had pituitary microadenoma (or no visible tumour), 24 were macroadenoma.

- $74(84 \%)$ patients had initial remission after surgery, during follow up 6 of those with initial remission had recurrence of CS.

\section{RESULTS}

- Those with initial remission had greater mean SD weight loss at 3 months (kg:-8.1 11.3 [initial remission, $\mathrm{n}=59$ ] v $0.8 \quad 8.8$ [non-remission, $\mathrm{n}=12$ ], $\mathrm{p}=0.007$ ) and at 6 months (kg:-14.5 12.1 [initial remission, $n=46$ ] v $-6.1 \quad 12.1$ [non-remission, $n=12$ ],

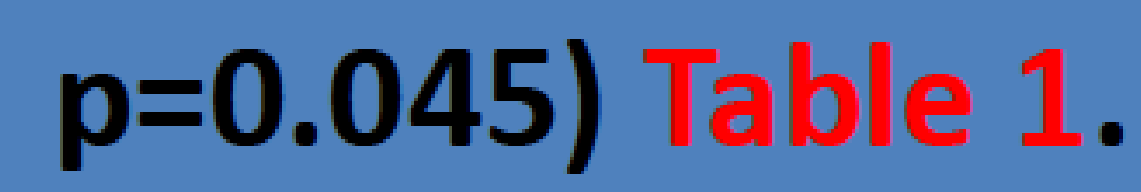

- There was evidence that less weight loss or weight gain at three $(p=0.002)$ and six $(p=0.014)$ months was associated with increased risk of relapse

- At most recent follow up, weight loss after TSS within the first $3(p=0.019)$ and weight loss within the first 6 months ( $p=0.035$ ) was also predictive of current remission status.

Weight change outcome by initial remission status

\begin{tabular}{|c|c|c|c|}
\hline & $\begin{array}{c}\text { No } \\
\text { Remission } \\
(\mathrm{N}=18)\end{array}$ & $\begin{array}{c}\text { Initial } \\
\text { Remission } \\
(\mathrm{N}=\mathbf{8 3})\end{array}$ & $\mathrm{p}$ valuc \\
\hline Weight Change: & & & 0.18 \\
\hline Missing & $4(\%)$ & $9(\%)$ & \\
\hline No Loss or Increase & $8(57.1 \%)$ & $28(37.8 \%)$ & \\
\hline Wgl Loss $(\geqslant 1 \mathrm{~kg} / \mathrm{mon})$. & $6(42.9 \%)$ & $46(62.2 \%)$ & \\
\hline Weight Change: 1 Month & & & 0.12 \\
\hline $\mathrm{N}$ & 13 & 68 & \\
\hline Mean (SD) & $0.4(4.7)$ & $-2.0(4.6)$ & \\
\hline Median & -0.5 & -0.9 & \\
\hline Range & $(-6.4-10.3)$ & $(-29.9-3.4)$ & \\
\hline Weight Change: 3 Months & & & 0.007 \\
\hline $\mathrm{N}$ & 12 & 59 & \\
\hline Mean (SD) & $0.8(8.8)$ & $-8.1(11.3)$ & \\
\hline Median & -1.2 & -5.0 & \\
\hline Range & $(-6.6-25.4)$ & $(-77.1-2.2)$ & \\
\hline Weight Change: 6 Months & & & 0.045 \\
\hline $\mathrm{N}$ & 12 & 46 & \\
\hline Mean (SD) & $-6.1(12.1)$ & $-14.5(12.1)$ & \\
\hline Median & 0.0 & -13.7 & \\
\hline Range & $(-26.0-9.8)$ & $(-76.3--0.3)$ & \\
\hline
\end{tabular}

Differences in weight loss over time between remission and non remission

\begin{tabular}{llccc}
\hline & & \multicolumn{3}{c}{ Hazard Ratio } \\
Variable & Time & Frequency & $(\mathbf{9 5 \%} \mathbf{C I})$ & Overall \\
Wgt Change & Month 1 & 81 & $1.14(0.99,1.29)$ & 0.056 \\
& Month 3 & 71 & $1.08(1.03,1.14)$ & 0.002 \\
& Month 6 & 58 & $1.08(1.02,1.15)$ & 0.014
\end{tabular}

\section{DISCUSSION}

- The current study demonstrates that the majority of patients with initial remission after TSS will experience weight loss. Patients with initial nonremission although to a lesser degree can also experience initial weight loss after TSS. The study also confirms that those with initial remission had greater weight loss at 3 and 6 months relative to those without initial remission. Weight changes within the first six months was also predictive of long term remission status.

\section{CONCLUSION}

- After TSS for CD, weight loss within the first six months maybe an additional clinical indicator associated with early biochemical and current remission status. 